\title{
Velocidade de crescimento de crianças de uma coorte até o sexto mês de vida
}

\author{
Growth rate of children of a cohort up to the sixth month of life
}

Anahi Cézar de Lima Lins (https://orcid.org/0000-0003-2983-0051) ${ }^{1}$

Dixis Figueroa Pedraza (http://orcid.org/0000-0002-5394-828X) ${ }^{1}$

\footnotetext{
${ }^{1}$ Universidade Estadual da Paraíba. R. das Baraúnas 351, Campus Universitário, Bodocongó. 58109-753 Campina Grande PB Brasil dixisfigueroa@gmail.com
}

\begin{abstract}
The scope of this article is to investigate factors that contributed to the growth rate of children from birth through the sixth month of life. It involves a cohort study with 144 children observed at birth and in the first, second and sixth months of life. Child head circumference $(\mathrm{cm})$ month), weight (g/day) and length ( $\mathrm{cm} /$ month) rates were compared according to birth, pregnancy, maternal and family characteristics and those related to the food, illness and health care of the child. The female sex and difficulty in breastfeeding contributed to the deceleration of all three growth rates, while maternal non-vaccination against tetanus affected the increase of head circumference and of child length. Severe maternal mental disorder, moderate/severe food insecurity, oatmeal consumption and failure to follow-up the development of the child influenced the head circumference and weight rates. The intake of alcohol during pregnancy, cesarean delivery, mixed/ predominant breastfeeding, use of infant formula and episodes of diarrhea/vomiting affected the head circumference, while inadequate prenatal care affected weight, and low-stature mothers and pacifier use affected length. Modifiable factors are highlighted, such as the consumption of oatmeal and infant formula, and the use of pacifiers, which are related to breastfeeding in infants.
\end{abstract}

Key words Growth, Breastfeeding, Infant
Resumo $O$ objetivo deste artigo é investigar fatores que contribuiram na velocidade de crescimento de crianças até o sexto mês de vida. Coorte com 144 crianças observadas ao nascimento e no primeiro, segundo e sexto mês de vida. As velocidades de perímetro cefálico ( $\mathrm{cm} / \mathrm{mês})$, peso (g/dia) e comprimento ( $\mathrm{cm} / \mathrm{mês})$ foram comparadas segundo características ao nascimento, da gravidez, maternas, da família e relacionadas à alimentação, adoecimento e cuidados de saúde da criança. $O$ sexo feminino e a dificuldade de amamentar contribuíram com a desaceleração das três velocidades de crescimento; a não vacinação materna contra o tétano, as velocidades de perímetro cefálico e comprimento. Transtorno mental materno grave, insegurança alimentar moderada/grave, consumo de mingau e o não acompanhamento da criança influenciaram as velocidades de perímetro cefálico e peso. Uso de bebida alcoólica durante a gravidez, parto cesariano, aleitamento materno misto/predominante, uso de fórmula infantil $e$ episódios de diarreia/vômito influenciaram a velocidade de perímetro cefálico; pré-natal inadequado, ade peso; mães baixinhas e uso chupeta, a de comprimento. Sobressaem fatores modificáveis como o consumo de mingau e de fórmula infantil e o uso de chupeta que têm em comum a relação com o aleitamento materno do lactente.

Palavras-chave Crescimento, Aleitamento materno, Lactente 


\section{Introdução}

O crescimento pós-natal sofre importante influência do potencial genético, do crescimento intrauterino e dos efeitos de uma série de fatores nutricionais, socioeconômicos, culturais e ambientais ${ }^{1,2}$. Nesse contexto, a má nutrição é responsável pelo surgimento de doenças infantis, apresentando ainda efeitos negativos no desenvolvimento infantil ${ }^{3}$. As práticas nutricionais adequadas no útero e nos primeiros mil dias de vida são essenciais para a criança crescer adequadamente e podem atenuar o efeito prejudicial de um crescimento fetal inadequado ${ }^{1,3}$. Além disso, o peso e o comprimento podem ser seriamente influenciados pela ocorrência de morbidades na fase de crescimento ${ }^{1}$.

O primeiro ano de vida, marcadamente os seis primeiros meses, caracteriza-se por ser a fase de maior velocidade de crescimento e acentuada vulnerabilidade a agravos nutricionais, tornando-o um período crítico ${ }^{4-6}$. Dessa forma, a Organização Mundial de Saúde (OMS) recomenda o consumo do leite materno de forma exclusiva até o sexto mês de vida por dispor de características nutricionais ideais para promover o crescimento nesta etapa da vida ${ }^{7}$.

O acompanhamento do crescimento físico configura um importante indicador de saúde desde a primeira infância até a puberdade. Este método pode ser avaliado por meio das medidas de peso, comprimento e perímetro cefálico, determinando o estado de saúde individual ${ }^{8}$. A avaliação dessas medidas ao longo do tempo, expresso como velocidade, representa a maneira ideal de descrever os padrões complexos de crescimento, permitindo avaliar de maneira mais precisa alterações no estado nutricional e suas nuances temporais ${ }^{4-6,8}$.

Avaliar o crescimento nos primeiros meses de vida e seus determinantes é essencial considerando sua importância para o acompanhamento clínico das crianças e no diagnóstico de condições que podem ser prevenidas. Ainda, por seus efeitos a médio e longo prazos, como no desenvolvimento neuropsicomotor e na prevenção de doenças ${ }^{7}$. Contudo, ainda são necessários estudos que investiguem a trajetória de crescimento das crianças ${ }^{4,8}$.

Diante do exposto, este estudo teve como objetivo investigar fatores que contribuíram na velocidade de crescimento de crianças no período do nascimento até os seis meses de vida.

\section{Métodos}

Trata-se de um estudo de coorte, desenvolvido entre primeiro de janeiro de 2018 e 31 de janeiro de 2019, com crianças acompanhadas do nascimento até seis meses de vida do Município de Mamanguape, Paraíba, região Nordeste do Brasil, distante em cerca de $60 \mathrm{~km}$ da cidade de João Pessoa, capital do estado. As crianças foram avaliadas ao nascimento e acompanhadas no $1^{\circ}$, $2^{\circ}$ e $6^{\circ}$ mês de vida, permitindo dentro da fase de maior velocidade de crescimento (seis primeiros meses) compreender avaliações nos momentos extremos, da fase neonatal e em etapas que abrangem os dois trimestres do período.

Foram convidadas a fazer parte do estudo, no momento do nascimento, as mães das crianças nascidas no Hospital Geral de Mamanguape no período de primeiro de janeiro a 31 de julho de 2018, residentes no município. O hospital dispõe de três salas de cirurgia, duas de parto e 15 leitos de maternidade. Na ala de obstetrícia da instituição, em pouco mais de três anos de atuação, houve 5.000 nascimentos, com apenas dois óbitos neonatais e taxa de $99 \%$ de partos seguros.

Segundo informações da diretoria do Hospital Geral de Mamanguape (hospital-maternidade), houve 335 nascimentos de mulheres residentes no município no período de duração da coleta de dados nessa instituição. Do total de 335 crianças elegíveis para o estudo, excluíram-se as que estavam em algum dos seguintes critérios: mãe de idade inferior aos 18 anos, má-formação congênita, gemelaridade, referenciadas para Unidade de Terapia Intensiva Neonatal, óbito neonatal, nascimento antes da $37^{\mathrm{a}}$ semana de gestação e peso ao nascer inferior a $2.500 \mathrm{~g}$.

A equipe responsável pela coleta de dados foi composta por acadêmicos de cursos de graduação da área de saúde e de pós-graduação em saúde pública. O controle de qualidade do estudo incluiu treinamento e padronização dos entrevistadores, construção de Manual de Instruções, realização de estudo piloto e supervisão do trabalho de campo. O estudo piloto foi conduzido na semana anterior de início da coleta de dados, no mesmo local onde foi realizada a coleta dos dados, com 13 crianças na mesma faixa etária e características das pertencentes ao estudo, as quais não foram incluídas nas análises.

Um membro da equipe do projeto ficou de plantão no hospital aguardando a internação das gestantes para a realização do convite à pesquisa 
e agendamento das próximas consultas. As crianças foram avaliadas ao nascimento, no hospital, e no $1^{\circ}, 2^{\circ}$ e $6^{\circ}$ mês de vida, no domicílio.

Ao nascimento da criança, foi aplicado às mães um questionário semiestruturado contendo informações sobre sexo da criança; idade materna; cuidados durante a gravidez (tempo de início e número de consultas do pré-natal, ingestão de bebida alcoólica e fumo, vacinação contra o tétano) e tipo de parto. Além disso, as mães foram medidas usando estadiômetro $\left(\mathrm{WCS}^{\circledR}\right)$ com escala em milímetros ( $\mathrm{mm}$ ) e responderam às perguntas da escala mundial de insegurança alimentar'. A baixa estatura materna foi definida pelo ponto de corte de $155,0 \mathrm{~cm}^{10}$. As famílias foram classificadas em segurança alimentar e nutricional quando todas as questões foram respondidas de forma negativa; insegurança alimentar e nutricional leve quando teve-se até duas questões com respostas positivas; insegurança alimentar e nutricional moderada nos casos de três a seis respostas positivas e insegurança alimentar e nutricional grave para sete ou oito respostas positivas ${ }^{9}$.

No $1^{\circ}, 2^{\circ}$ e $6^{\circ}$ mês de vida da criança, foram obtidas informações sobre a dificuldade de amamentar, as práticas alimentares (consumo ou não de leite materno, leite de vaca, mingau, água/chá e fórmula infantil), o uso de chupeta e a presença de episódios de diarreia/vômito. Para a classificação do tipo de aleitamento materno, utilizaramse as definições da OMS, recomendadas pelo Ministério da Saúde, que classifica em aleitamento materno exclusivo, predominante, misto ou parcial e complementado ${ }^{11}$. Para fins de análise, consideraram-se as categorias aleitamento materno exclusivo/predominante e aleitamento materno misto/artificial. Também foi investigado em todas as avaliações o preenchimento das curvas de crescimento da Caderneta de Saúde da Criança (CSC), considerando-se inadequado quando não houve nenhum registro no período.

Para aferição da saúde mental materna seis meses após o parto, as mães responderam a versão validada e adaptada para o português do $G e$ neral Health Questionnaire com 12 itens (GHQ$12)^{12}$. Foram estabelecidas duas variáveis para avaliar a saúde mental materna a partir deste instrumento: transtornos mentais comuns (definida a partir do ponto de corte de pelo menos três respostas positivas nos 12 potenciais) e transtornos mentais graves (identificada pelo ponto de corte de pelo menos cinco respostas positivas) ${ }^{13}$.

Em todas as avaliações (ao nascimento, no $1^{\circ}, 2^{\circ}$ e $6^{\circ}$ mês) as medidas de perímetro cefálico, peso e comprimento das crianças foram mensu- radas por entrevistadores previamente treinados, seguindo técnicas padronizadas ${ }^{14}$. Para o perímetro cefálico foi usada fita métrica inelástica de precisão de $1 \mathrm{~mm}$. O peso foi mensurado utilizando-se balança eletrônica e digital, pediátrica, com capacidade de $15 \mathrm{~kg}$ e precisão de 10 gramas, sempre sem roupas ou fralda. O comprimento foi aferido com a criança despida, utilizando um antropômetro infantil de madeira, com régua graduada de 0 a $100 \mathrm{~cm}$, precisão de $1 \mathrm{~mm}$. Todas as medidas foram realizadas duas vezes e a média usada para fins de registro. Crianças que nasceram com o peso maior que 2.500 e menor que 3.000 gramas foram consideradas com peso insuficiente ao nascer, e maior ou igual que 3.000 gramas com peso satisfatório ${ }^{15}$. Essa informação foi incluída dentre as variáveis de nascimento.

As velocidades de perímetro cefálico $(\mathrm{cm} /$ mês), peso (g/dia) e comprimento ( $\mathrm{cm} / \mathrm{mês})$ das crianças foram calculadas do nascimento até o $1^{\circ}, 2^{\circ}$ e $6^{\circ}$ mês, através dos seguintes cálculos:

Velocidade de perímetro cefálico $(\mathrm{cm} / \mathrm{mês})=$ (perímetro cefálico no $1^{\circ}, 2^{\circ}$ e $6^{\circ}$ mês - perímetro cefálico ao nascer) / idade em meses

Velocidade de peso $(\mathrm{g} / \mathrm{dia})=\left(\right.$ peso no $1^{\circ}, 2^{\circ} \mathrm{e}$ $6^{\circ}$ mês - peso ao nascer) / idade em dias

Velocidade de comprimento $(\mathrm{cm} / \mathrm{mês})=$ (comprimento no $1^{\circ}, 2^{\circ}$ e $6^{\circ}$ mês - comprimento ao nascer) / idade em meses

A digitação dos dados foi realizada em dupla entrada por digitadores treinados em banco customizado com verificações de consistência e restrições de intervalo, desenvolvido no Microsoft Office Excel. Para fins de análise, utilizou-se o banco construído após verificação de inconsistências.

Tendo em vista que não foi previsto o tamanho amostral inicial para avaliar a relação entre as velocidades de crescimento e as variáveis de interesse, foi calculado o poder da amostra a posteriori para comparar médias em todos os meses avaliados, com o intervalo de confiança de $95 \%$. Considerando a diferença entre as médias de velocidade de perímetro cefálico, o poder variou de $77,9 \%$ a $99,7 \%$. Para a diferença entre a velocidade de peso, o poder variou de $88,7 \%$ a $99,6 \%$. No caso da velocidade de comprimento, a variação foi de $84,8 \%$ a $99,9 \%$. Os cálculos foram conduzidos no programa OpenEpi.

As variáveis obtidas ao nascimento foram comparadas conforme o acompanhamento completo ou não (perdas de seguimento) da criança até o $6^{\circ}$ mês de vida, para avaliação de possível viés de seleção relacionado às perdas de seguimento. Para essa comparação, foi utilizado o teste 
Qui-quadrado de Pearson e o teste T de Student, no programa estatístico Stata, versão 10.0.

A distribuição das variáveis velocidade de perímetro cefálico, velocidade de peso e velocidade de comprimento foram analisadas por meio do teste Shapiro Wilk, em todos os momentos. Para análise das diferenças entre os valores das velocidades de crescimento e as variáveis explicativas (sexo, idade materna, estatura materna, presença de transtorno mental comum materno, presença de transtorno mental grave materno, trimestre de início do pré-natal, número de consultas de pré-natal, ingestão de bebida alcoólica durante a gravidez, fumo durante a gravidez, vacinação contra o tétano durante a gravidez, tipo de parto, peso ao nascer, segurança alimentar e nutricional, dificuldade para amamentar, tipo de aleitamento materno, consumo de leite de vaca, consumo de mingau, consumo de água/chá, consumo de fórmula infantil, uso de chupeta, presença de episódios de diarreia/vômito e preenchimento das curvas de crescimento da CSC) foi utilizado o teste T de Student. As variáveis foram ajustadas, estimando-se o nível de significância segundo o teste de heterogeneidade de Wald. Foi utilizada uma estratégia passo a passo iniciando com um modelo com todas as variáveis ajustadas, sendo retiradas aquelas que tiveram valor de $\mathrm{p}$ igual ou menor que 0,20 ao utilizar o teste T de Student. O nível de rejeição para a hipótese de nulidade foi de $\mathrm{p}<0,05$. Todas as análises estatísticas foram conduzidas no programa estatístico Stata, versão 10.0 .

Do ponto de vista normativo, o projeto está em conformidade com a Resolução 466/2012 do Conselho Nacional de Saúde e foi aprovado pelo Comitê de Ética em Pesquisa da Universidade Estadual da Paraíba. As mães das crianças assinaram o Termo de Consentimento Livre e Esclarecido (TCLE) e foram devidamente orientadas de todos os procedimentos, objetivos e vantagens na sua participação. Crianças que apresentaram durante a coorte curvas de crescimento indicativas de alterações nos padrões de crescimento de normalidade foram encaminhadas para os serviços de saúde com fim de tratamento.

\section{Resultados}

Das 335 crianças nascidas no Hospital Geral de Mamanguape de mães residentes no município no período de primeiro de janeiro a 31 de julho de 2018, 95 foram excluídas e 35 mães não aceitaram participar da pesquisa. Do total de crian- ças que iniciaram o estudo $(\mathrm{n}=205), 12$ foram perdidas no $1^{\circ}$ mês, 22 no $2^{\circ}$ e 27 no $6^{\circ}$, o que permitiu completar a avaliação de 193, 171 e 144 casos nesses mesmos períodos. O fluxograma de seleção da população de estudo pode ser visualizado na Figura 1.

A Tabela 1 mostra a comparação das características ao nascimento das crianças que completaram o acompanhamento e das crianças perdidas durante o seguimento até o $6^{\circ}$ mês. A ausência de diferença estatística entre os grupos $(\mathrm{p}<0,05)$ é indicativa de não ter acontecido viés de seleção devido às perdas de seguimento.

Das 144 crianças avaliadas até os seis meses, $56,3 \%$ eram meninos. Quanto ao nascimento, em 18,8\% foi por parto cesáreo e 18,1\% com peso insuficiente. Em relação às práticas alimentares, observou-se que o aleitamento materno exclusivo/predominante de $53,2 \%$ e $50,0 \%$ no $1^{\circ}$ e $2^{\circ}$ mês, respectivamente, passou para $6,6 \%$ no $6^{\circ}$ mês. A frequência de consumo de mingau foi de $23,4 \%, 32,2 \%$ e $58,5 \%$ no $1^{\circ}, 2^{\circ}$ e $6^{\circ}$ mês, respectivamente, enquanto de fórmula infantil foi de $45,4 \%, 47,2 \%$ e $72,6 \%$, nesses mesmos períodos. A dificuldade de amamentar oscilou de $26,3 \%$, no $1^{\circ}$ mês, para $80,0 \%$, no $6^{\circ}$ mês, e o uso de chupeta de $43,3 \%$, no primeiro momento, para 51,1\%, no último momento. A referência materna sobre presença de episódios de diarreia/ vômito na criança foi de $12,1 \%$, no mês $1,5,7 \%$, no mês 2, e 72,6\%, no mês 6 .

A velocidade de crescimento do perímetro cefálico dos lactentes nas três avaliações, de acordo com as variáveis de interesse, encontra-se na Tabela 2. Observou-se maior velocidade no sexo masculino, nas três avaliações, havendo redução do ganho de perímetro cefálico a partir do $2^{\circ}$ mês em ambos os sexos. Também houve maior velocidade de crescimento nos três momentos entre as crianças cujas mães receberam a vacinação contra o tétano durante a gravidez, em situação de segurança alimentar/insegurança alimentar leve e nos casos em que não foi diagnosticado transtorno mental grave materno. Ainda, a não ingestão de bebida alcoólica durante a gestação, o parto normal, a facilidade de amamentar, o aleitamento materno exclusivo/predominante, o não consumo de mingau e de fórmula infantil, a ausência de episódios de diarreia/vômito e o preenchimento da curva de crescimento de perímetro cefálico na CSC contribuíram com maiores ganhos de perímetro cefálico em algum momento do seguimento.

Para a velocidade de peso (Tabela 3), também se observou maiores ganhos nos meninos do que 
335 nascimentos de mães residentes no Município de Mamanguapeno período de $1^{\circ}$ de janeiro a 31 de julho de 2018

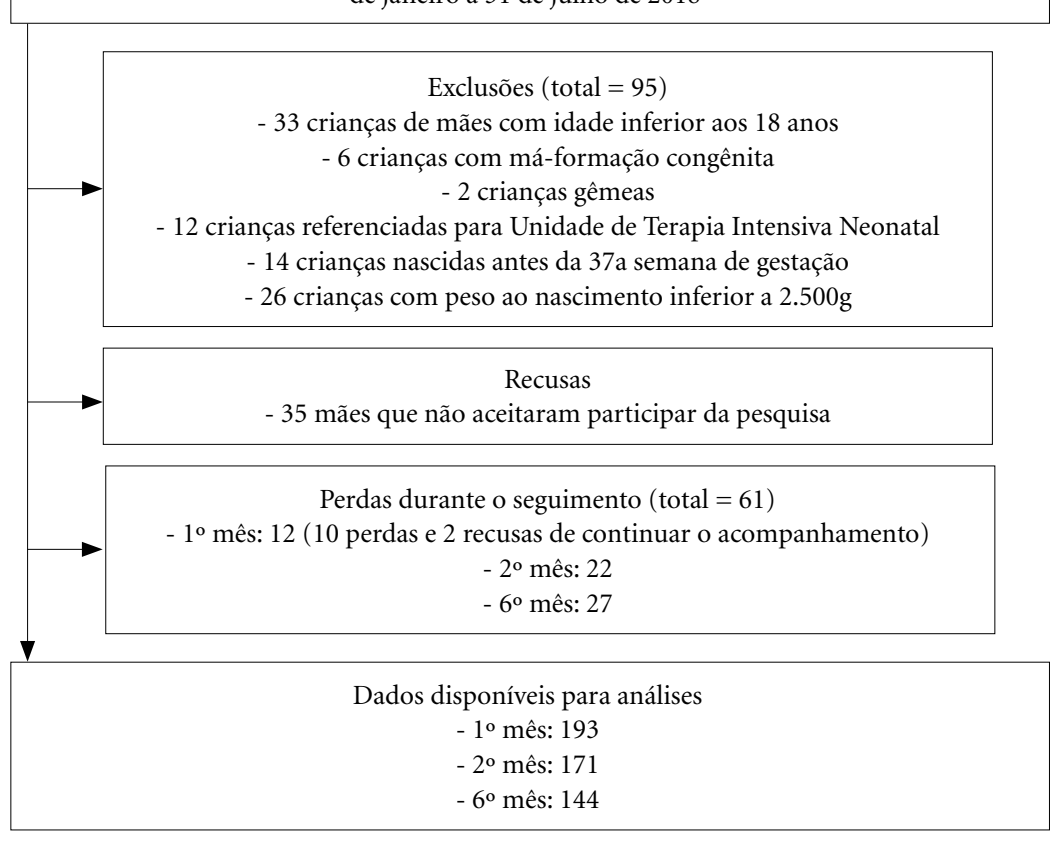

Figura 1. Fluxograma de seleção da população de estudo para analisar o crescimento de crianças até o sexto mês de vida. Mamanguape, Paraíba, 2018.

Fonte: Elaborado pelos autores.

nas meninas, nas três avaliações. Maiores médias de ganho de peso ocorreram entre as crianças de mães que iniciaram o pré-natal no primeiro trimestre da gestação e entre aquelas que realizaram $\geq 6$ consultas de pré-natal, em todas as avaliações. Crianças em situação de segurança alimentar/ insegurança alimentar leve tiveram ganhos mais expressivos no $1^{\circ}$ e no $2^{\circ}$ mês, enquanto no $1^{\circ}$ mês o resultado foi similar também nos lactentes de mães sem diagnóstico de transtorno mental grave, que não vivenciaram dificuldades de amamentação, que não consumiram mingau e que apresentaram a CSC com anotação na curva de crescimento de peso.

No $6^{\circ}$ mês de vida das crianças, observou-se maior velocidade de comprimento nos meninos. A vacinação contra o tétano durante a gestação, no $1^{\circ}$ mês, a ausência de dificuldade para amamentar, no $1^{\circ}$ e $2^{\circ}$ mês, e o não uso de chupeta, no $6^{\circ}$ mês, também representaram maiores ganhos de comprimento. Nas três avaliações, a velocidade de comprimento foi maior entre os lactentes de mães com estatura adequada (Tabela 4).

Após o ajuste das variáveis, foram confirmadas as associações estatísticas descritas nas
Tabelas 2, 3 e 4 . O único resultado diferente constatado foi para o não consumo de mingau, característica que ganhou significância estatística para as velocidades de perímetro cefálico e peso, no mês 6 e 2 , respectivamente.

\section{Discussão}

Este estudo se propôs a avaliar a velocidade de crescimento de crianças nos primeiros seis meses de vida, segundo o sexo e variáveis ao nascimento da criança, cuidados durante a gravidez, características maternas e da família, e aspectos relacionados à alimentação, ao adoecimento $\mathrm{e}$ aos cuidados de saúde da criança. Encontraramse resultados pouco comuns na literatura que os tornam relevantes à compreensão das condições associadas ao crescimento infantil.

Para todos os indicadores antropométricos, a velocidade de crescimento diminuiu gradativamente até o $6^{\circ}$ mês em ambos os sexos, independentemente do tipo de aleitamento materno ofertado, corroborando as observações de estudos anteriores ${ }^{4,16}$. Esse comportamento reflete a 
Tabela 1. Características das crianças ao nascimento conforme o acompanhamento completo ou não (perdas de seguimento) até o sexto mês de vida. Mamanguape, Paraíba, 2018.

\begin{tabular}{|c|c|c|c|}
\hline \multirow[t]{2}{*}{ Características da amostra } & $\begin{array}{c}\text { Crianças com } \\
\text { acompanhamento } \\
\text { completo } \\
(n=144)\end{array}$ & $\begin{array}{c}\text { Crianças com } \\
\text { acompanhamento } \\
\text { incompleto/perdas } \\
\text { de seguimento } \\
(n=61)\end{array}$ & \multirow[t]{2}{*}{ p-valor } \\
\hline & $\%(n)$ & $\%(n)$ & \\
\hline \multicolumn{4}{|l|}{ Sexo da criança } \\
\hline Masculino & $68,1(81)$ & $31,9(38)$ & 0,423 \\
\hline Feminino & $73,3(63)$ & $26,7(23)$ & \\
\hline \multicolumn{4}{|l|}{ Idade materna (anos) } \\
\hline $18-34$ & $69,4(100)$ & $30,6(44)$ & 0,903 \\
\hline$>34$ & $72,1(44)$ & $27,9(17)$ & \\
\hline \multicolumn{4}{|l|}{ Estatura materna $(\mathrm{cm})$} \\
\hline Adequada & $70,8(109)$ & $29,2(45)$ & 0,561 \\
\hline Baixa estatura $(<155)$ & $68,6(35)$ & $31,4(16)$ & \\
\hline \multicolumn{4}{|l|}{ Início do pré-natal } \\
\hline $1^{\circ}$ trimestre & $71,9(115)$ & $28,1(45)$ & 0,335 \\
\hline Após $1^{\circ}$ trimestre & $64,4(29)$ & $35,6(16)$ & \\
\hline \multicolumn{4}{|l|}{$\mathrm{N}^{\circ}$ de consultas de pré-natal } \\
\hline$\geq 6$ & $71,9(120)$ & $28,1(47)$ & 0,290 \\
\hline$<6$ & $63,2(24)$ & $36,8(14)$ & \\
\hline \multicolumn{4}{|l|}{ Ingestão de bebida alcoólica durante a gravidez } \\
\hline Não & $70,0(133)$ & $30,0(57)$ & 0,786 \\
\hline Sim & $73,3(11)$ & $26,7(4)$ & \\
\hline \multicolumn{4}{|l|}{ Fumo durante a gravidez } \\
\hline Não & $70,1(138)$ & $29,9(59)$ & 0,764 \\
\hline Sim & $75,0(6)$ & $25,0(2)$ & \\
\hline \multicolumn{4}{|l|}{ Vacinação contra o tétano durante a gravidez } \\
\hline Sim & $71,1(138)$ & $28,9(56)$ & 0,317 \\
\hline Não & $55,6(5)$ & $44,4(4)$ & \\
\hline \multicolumn{4}{|l|}{ Tipo de parto } \\
\hline Normal & $72,7(117)$ & $27,3(44)$ & 0,146 \\
\hline Cesárea & $61,4(27)$ & $38,6(17)$ & \\
\hline \multicolumn{4}{|l|}{ Peso ao nascer $(\mathrm{g})$} \\
\hline Satisfatório $(\geq 3.000)$ & $69,8(118)$ & $30,2(51)$ & 0,775 \\
\hline Insuficiente (2.500-2.999) & $72,2(26)$ & $27,8(10)$ & \\
\hline \multicolumn{4}{|l|}{ Segurança alimentar e nutricional } \\
\hline Segurança alimentar/insegurança alimentar leve & $72,8(115)$ & $27,2(43)$ & 0,145 \\
\hline Insegurança alimentar moderada/grave & $61,7(29)$ & $38,3(18)$ & \\
\hline \multicolumn{4}{|l|}{ Perímetro cefálico ao nascer $(\mathrm{cm})$} \\
\hline Média $\pm \mathrm{DP}$ & $34,1 \pm 1,6$ & $34,3 \pm 1,5$ & 0,817 \\
\hline \multicolumn{4}{|l|}{ Peso ao nascer (g) } \\
\hline Média $\pm D P$ & $3439,8 \pm 479,1$ & $3535,9 \pm 470,7$ & 0,506 \\
\hline \multicolumn{4}{|l|}{ Comprimento ao nascer $(\mathrm{cm})$} \\
\hline Média $\pm \mathrm{DP}$ & $49,5 \pm 2,1$ & $49,8 \pm 2,2$ & 0,799 \\
\hline
\end{tabular}

Fonte: Elaborado pelos autores.

variabilidade temporal nos parâmetros antropométricos que se inicia com uma fase de acelera- ção prosseguida pela desaceleração ${ }^{17,18}$. A desaceleração do ganho de peso, por exemplo, constitui 
Tabela 2. Velocidade de perímetro cefálico de crianças até o sexto mês de vida segundo sexo, características maternas, cuidados durante a gravidez, variáveis de nascimento, segurança alimentar e nutricional, dificuldade para amamentar, práticas alimentares, uso de chupeta, presença de episódios de diarreia/vômito, saúde mental materna e vigilância do crescimento. Mamanguape, Paraíba, 2018.

\begin{tabular}{|c|c|c|c|c|c|c|}
\hline \multirow{3}{*}{ Variáveis } & \multicolumn{6}{|c|}{ Velocidade de perímetro cefálico $(\mathrm{cm} / \mathrm{mês})$} \\
\hline & \multicolumn{2}{|c|}{ Mês 1} & \multicolumn{2}{|c|}{ Mês 2} & \multicolumn{2}{|c|}{ Mês 6} \\
\hline & Média $\pm \mathrm{DP}$ & p-valor ${ }^{*}$ & Média $\pm \mathrm{DP}$ & p-valor ${ }^{*}$ & Média $\pm \mathrm{DP}$ & p-valor ${ }^{\star}$ \\
\hline \multicolumn{7}{|l|}{ Sexo da criança } \\
\hline Masculino & $4,0 \pm 1,6$ & 0,041 & $2,9 \pm 0,8$ & 0,030 & $1,6 \pm 0,3$ & 0,008 \\
\hline Feminino & $3,6 \pm 1,4$ & & $2,6 \pm 0,8$ & & $1,5 \pm 0,3$ & \\
\hline \multicolumn{7}{|l|}{ Idade materna (anos) } \\
\hline $18-34$ & $3,8 \pm 1,5$ & 0,288 & $2,8 \pm 0,8$ & 0,346 & $1,6 \pm 0,2$ & 0,129 \\
\hline$>34$ & $3,6 \pm 1,3$ & & $2,7 \pm 0,7$ & & $1,5 \pm 0,3$ & \\
\hline \multicolumn{7}{|l|}{ Estatura materna $(\mathrm{cm})$} \\
\hline Adequada & $4,0 \pm 1,4$ & 0,171 & $2,9 \pm 0,7$ & 0,053 & $1,6 \pm 0,3$ & 0,114 \\
\hline Baixa estatura $(<155)$ & $3,7 \pm 1,6$ & & $2,6 \pm 0,8$ & & $1,5 \pm 0,3$ & \\
\hline \multicolumn{7}{|c|}{ Transtorno Mental Comum materno } \\
\hline Não & $4,1 \pm 1,1$ & 0,099 & $2,9 \pm 0,6$ & 0,161 & $1,6 \pm 0,3$ & 0,082 \\
\hline Sim & $3,7 \pm 1,6$ & & $2,7 \pm 0,8$ & & $1,5 \pm 0,3$ & \\
\hline \multicolumn{7}{|c|}{ Transtorno Mental Grave materno } \\
\hline Não & $4,1 \pm 1,4$ & 0,037 & $2,9 \pm 0,8$ & 0,044 & $1,6 \pm 0,3$ & 0,013 \\
\hline Sim & $3,6 \pm 1,5$ & & $2,6 \pm 0,8$ & & $1,5 \pm 0,3$ & \\
\hline \multicolumn{7}{|l|}{ Início do pré-natal } \\
\hline $1^{\circ}$ trimestre & $3,8 \pm 1,6$ & 0,500 & $2,7 \pm 0,8$ & 0,558 & $1,5 \pm 0,3$ & 0,635 \\
\hline Após $1^{\circ}$ trimestre & $3,8 \pm 1,3$ & & $2,8 \pm 0,8$ & & $1,6 \pm 0,3$ & \\
\hline \multicolumn{7}{|l|}{$\mathrm{N}^{\circ}$ de consultas de pré-natal } \\
\hline$\geq 6$ & $3,8 \pm 1,6$ & 0,507 & $2,7 \pm 0,8$ & 0,517 & $1,6 \pm 0,3$ & 0,412 \\
\hline$<6$ & $3,8 \pm 1,3$ & & $2,8 \pm 0,7$ & & $1,5 \pm 0,3$ & \\
\hline \multicolumn{7}{|c|}{$\begin{array}{l}\text { Ingestão de bebida alcoólica durante } \\
\text { a gravidez }\end{array}$} \\
\hline Não & $3,9 \pm 1,5$ & 0,017 & $2,8 \pm 0,8$ & 0,045 & $1,6 \pm 0,3$ & 0,111 \\
\hline Sim & $2,8 \pm 1,0$ & & $2,3 \pm 0,5$ & & $1,4 \pm 0,1$ & \\
\hline \multicolumn{7}{|l|}{ Fumo durante a gravidez } \\
\hline Não & $3,8 \pm 1,5$ & 0,058 & $2,8 \pm 0,8$ & 0,194 & $1,6 \pm 0,3$ & 0,312 \\
\hline Sim & $2,8 \pm 1,3$ & & $2,5 \pm 0,6$ & & $1,5 \pm 0,2$ & \\
\hline \multicolumn{7}{|c|}{$\begin{array}{l}\text { Vacinação contra o tétano durante a } \\
\text { gravidez }\end{array}$} \\
\hline Sim & $4,9 \pm 2,7$ & 0,041 & $3,3 \pm 0,7$ & 0,032 & $1,8 \pm 0,4$ & 0,026 \\
\hline Não & $3,6 \pm 1,5$ & & $2,2 \pm 0,8$ & & $1,5 \pm 0,3$ & \\
\hline \multicolumn{7}{|l|}{ Tipo de parto } \\
\hline Normal & $3,9 \pm 1,4$ & 0,049 & $2,8 \pm 0,8$ & 0,035 & $1,6 \pm 0,3$ & 0,109 \\
\hline Cesárea & $3,2 \pm 1,9$ & & $2,5 \pm 0,9$ & & $1,5 \pm 0,3$ & \\
\hline
\end{tabular}

um fenômeno fisiológico natural que não deve ser confundido com uma falha no crescimento, o que poderia levar à introdução precoce de alimentos complementares em crianças saudáveis e com bom ritmo de crescimento ${ }^{16}$.

No Maranhão, ao analisar os dados de perímetro cefálico do nascimento ao $6^{\circ}$ mês de vida, pesquisadores apontaram melhor situação nos meninos do que nas meninas ${ }^{19}$, o que foi confirmado no estudo em tela nos três momentos do acompanhamento. Do mesmo modo que o tamanho corporal, o desenvolvimento muscular e esquelético, e a distribuição de gordura, o perímetro cefálico constitui uma característica sexual 
Tabela 2. Velocidade de perímetro cefálico de crianças até o sexto mês de vida segundo sexo, características maternas, cuidados durante a gravidez, variáveis de nascimento, segurança alimentar e nutricional, dificuldade para amamentar, práticas alimentares, uso de chupeta, presença de episódios de diarreia/vômito, saúde mental materna e vigilância do crescimento. Mamanguape, Paraíba, 2018.

\begin{tabular}{|c|c|c|c|c|c|c|}
\hline \multirow{3}{*}{ Variáveis } & \multicolumn{6}{|c|}{ Velocidade de perímetro cefálico (cm/mês) } \\
\hline & \multicolumn{2}{|c|}{ Mês 1} & \multicolumn{2}{|c|}{ Mês 2} & \multicolumn{2}{|c|}{ Mês 6} \\
\hline & Média \pm DP & p-valor* & Média \pm DP & p-valor* & Média \pm DP & p-valor ${ }^{\star}$ \\
\hline \multicolumn{7}{|l|}{ Peso ao nascer $(\mathrm{g})$} \\
\hline Satisfatório $(\geq 3.000)$ & $3,7 \pm 1,5$ & 0,894 & $2,7 \pm 0,8$ & 0,985 & $1,5 \pm 0,3$ & 0,969 \\
\hline Insuficiente (2.500-2.999) & $4,1 \pm 1,7$ & & $3,1 \pm 0,8$ & & $1,7 \pm 0,3$ & \\
\hline \multicolumn{7}{|l|}{ Segurança alimentar e nutricional } \\
\hline $\begin{array}{l}\text { Segurança alimentar/insegurança } \\
\text { alimentar leve }\end{array}$ & $3,9 \pm 1,5$ & 0,048 & $2,8 \pm 0,8$ & 0,036 & $1,6 \pm 0,3$ & 0,031 \\
\hline $\begin{array}{l}\text { Insegurança alimentar moderada/ } \\
\text { grave }\end{array}$ & $3,0 \pm 1,6$ & & $2,5 \pm 0,8$ & & $1,5 \pm 0,3$ & \\
\hline \multicolumn{7}{|l|}{ Dificuldade para amamentar } \\
\hline Não & $4,0 \pm 1,5$ & 0,022 & $2,8 \pm 0,8$ & 0,364 & $1,5 \pm 0,3$ & 0,569 \\
\hline Sim & $3,4 \pm 1,5$ & & $2,7 \pm 0,5$ & & $1,6 \pm 0,3$ & \\
\hline \multicolumn{7}{|l|}{ Tipo de aleitamento materno } \\
\hline Exclusivo/predominante & $3,9 \pm 1,4$ & 0,109 & $3,0 \pm 0,8$ & 0,046 & $1,6 \pm 0,3$ & 0,235 \\
\hline Misto/artificial & $3,6 \pm 1,5$ & & $2,5 \pm 0,7$ & & $1,5 \pm 0,3$ & \\
\hline \multicolumn{7}{|l|}{ Consumo de leite de vaca } \\
\hline Não & $3,8 \pm 1,5$ & 0,262 & $2,6 \pm 0,5$ & 0,236 & $1,6 \pm 0,3$ & 0,084 \\
\hline $\operatorname{Sim}$ & $3,4 \pm 1,7$ & & $2,8 \pm 0,8$ & & $1,5 \pm 0,3$ & \\
\hline \multicolumn{7}{|l|}{ Consumo de mingau } \\
\hline Não & $3,9 \pm 1,5$ & 0,020 & $2,8 \pm 0,8$ & 0,237 & $1,6 \pm 0,3$ & 0,080 \\
\hline Sim & $3,3 \pm 1,5$ & & $2,7 \pm 0,8$ & & $1,5 \pm 0,2$ & \\
\hline \multicolumn{7}{|l|}{ Consumo de água/chá } \\
\hline Não & $3,9 \pm 1,4$ & 0,271 & $2,8 \pm 0,8$ & 0,321 & $1,7 \pm 0,5$ & 0,241 \\
\hline $\operatorname{Sim}$ & $3,7 \pm 1,6$ & & $2,7 \pm 0,8$ & & $1,5 \pm 0,3$ & \\
\hline \multicolumn{7}{|l|}{ Consumo de fórmula infantil } \\
\hline Não & $4,0 \pm 1,5$ & 0,043 & $2,8 \pm 0,8$ & 0,117 & $1,6 \pm 0,3$ & 0,085 \\
\hline $\operatorname{Sim}$ & $3,6 \pm 1,5$ & & $2,7 \pm 0,8$ & & $1,5 \pm 0,3$ & \\
\hline \multicolumn{7}{|l|}{ Uso de chupeta } \\
\hline Não & $3,8 \pm 1,5$ & 0,250 & $2,8 \pm 0,8$ & 0,130 & $1,5 \pm 0,3$ & 0,559 \\
\hline $\operatorname{Sim}$ & $3,7 \pm 1,6$ & & $2,7 \pm 0,8$ & & $1,6 \pm 0,3$ & \\
\hline \multicolumn{7}{|l|}{ Diarreia/Vômito } \\
\hline Não & $3,9 \pm 1,5$ & 0,044 & $2,8 \pm 0,8$ & 0,269 & $1,6 \pm 0,3$ & 0,082 \\
\hline Sim & $3,2 \pm 1,4$ & & $2,6 \pm 0,3$ & & $1,5 \pm 0,3$ & \\
\hline \multicolumn{7}{|l|}{$\begin{array}{l}\text { Preenchimento do perímetro cefálico } \\
\text { na Caderneta de Saúde da Criança }\end{array}$} \\
\hline Sim & $4,2 \pm 1,6$ & 0,219 & $3,2 \pm 0,7$ & 0,016 & $1,6 \pm 0,2$ & 0,271 \\
\hline Não & $3,8 \pm 1,5$ & & $2,7 \pm 0,8$ & & $1,5 \pm 0,3$ & \\
\hline
\end{tabular}

土DP: desvio-padrão; ${ }^{\star}$ Teste T de Student.

Fonte: Elaborado pelos autores.

secundária que faz parte do dimorfismo sexual ${ }^{17}$. Adicionalmente, sugere-se que os níveis fetais de testosterona influenciam regiões cerebrais específicas que serão desenvolvidas na substância cinzenta sexualmente dimórfica ${ }^{20}$.
O maior ganho de peso até os seis meses de idade nas crianças do sexo masculino, conforme observado nesse estudo em todos os momentos do seguimento, reforça resultados prévios ${ }^{4,5,8}$. No que se refere ao ganho de comprimento, a melhor 
Tabela 3. Velocidade de peso de crianças até o sexto mês de vida segundo sexo, características maternas, cuidados durante a gravidez, variáveis de nascimento, segurança alimentar e nutricional, dificuldade para amamentar, práticas alimentares, uso de chupeta, presença de episódios de diarreia/vômito, saúde mental materna e vigilância do crescimento. Mamanguape, Paraíba, 2018.

\begin{tabular}{|c|c|c|c|c|c|c|}
\hline \multirow{3}{*}{ Variáveis } & \multicolumn{6}{|c|}{ Velocidade de peso (g/dia) } \\
\hline & \multicolumn{2}{|c|}{ Mês 1} & \multicolumn{2}{|c|}{ Mês 2} & \multicolumn{2}{|c|}{ Mês 6} \\
\hline & Média \pm DP & p-valor* & Média \pm DP & p-valor* & Média \pm DP & p-valor ${ }^{*}$ \\
\hline \multicolumn{7}{|l|}{ Sexo da criança } \\
\hline Masculino & $45,9 \pm 17,9$ & 0,044 & $41,4 \pm 9,8$ & 0,000 & $28,0 \pm 5,9$ & 0,000 \\
\hline Feminino & $41,0 \pm 15,5$ & & $35,2 \pm 9,2$ & & $24,4 \pm 5,6$ & \\
\hline \multicolumn{7}{|l|}{ Idade materna (anos) } \\
\hline $18-34$ & $46,9 \pm 15,5$ & 0,147 & $38,7 \pm 10,5$ & 0,478 & $26,5 \pm 6,3$ & 0,449 \\
\hline$>34$ & $43,1 \pm 17,3$ & & $38,6 \pm 6,7$ & & $26,2 \pm 4,7$ & \\
\hline \multicolumn{7}{|l|}{ Estatura materna $(\mathrm{cm})$} \\
\hline Adequada & $43,8 \pm 16,3$ & 0,447 & $39,0 \pm 9,8$ & 0,333 & $26,8 \pm 5,7$ & 0,128 \\
\hline Baixa estatura $(<155)$ & $43,4 \pm 18,8$ & & $38,2 \pm 10,5$ & & $25,5 \pm 6,8$ & \\
\hline \multicolumn{7}{|c|}{ Transtorno Mental Comum materno } \\
\hline Não & $43,4 \pm 17,1$ & 0,057 & $38,7 \pm 10,7$ & 0,465 & $26,5 \pm 5,8$ & 0,394 \\
\hline Sim & $38,1 \pm 13,3$ & & $38,5 \pm 10,7$ & & $26,1 \pm 7,0$ & \\
\hline \multicolumn{7}{|c|}{ Transtorno Mental Grave materno } \\
\hline Não & $45,7 \pm 16,2$ & 0,012 & $38,8 \pm 10,0$ & 0,449 & $26,9 \pm 6,0$ & 0,223 \\
\hline Sim & $39,0 \pm 16,9$ & & $38,6 \pm 10,3$ & & $26,1 \pm 6,1$ & \\
\hline \multicolumn{7}{|l|}{ Início do pré-natal } \\
\hline $1^{\circ}$ trimestre & $45,3 \pm 16,3$ & 0,017 & $39,7 \pm 9,9$ & 0,013 & $26,9 \pm 5,8$ & 0,032 \\
\hline Após $1^{\circ}$ trimestre & $37,8 \pm 18,7$ & & $35,0 \pm 9,6$ & & $24,5 \pm 6,6$ & \\
\hline \multicolumn{7}{|l|}{$\mathrm{N}^{\circ}$ de consultas de pré-natal } \\
\hline$\geq 6$ & $44,7 \pm 16,3$ & 0,046 & $39,3 \pm 9,4$ & 0,049 & $27,1 \pm 5,5$ & 0,001 \\
\hline$<6$ & $36,8 \pm 20,1$ & & $35,6 \pm 12,5$ & & $22,5 \pm 7,6$ & \\
\hline \multicolumn{7}{|c|}{$\begin{array}{l}\text { Ingestão de bebida alcoólica durante } \\
\text { a gravidez }\end{array}$} \\
\hline Não & $43,7 \pm 17,0$ & 0,595 & $38,9 \pm 10,1$ & 0,199 & $26,5 \pm 6,1$ & 0,264 \\
\hline Sim & $45,0 \pm 18,5$ & & $36,1 \pm 8,3$ & & $25,3 \pm 4,7$ & \\
\hline \multicolumn{7}{|l|}{ Fumo durante a gravidez } \\
\hline Não & $44,1 \pm 17,0$ & 0,108 & $38,9 \pm 10,0$ & 0,132 & $26,5 \pm 6,1$ & 0,177 \\
\hline Sim & $34,5 \pm 17,4$ & & $34,2 \pm 10,3$ & & $24,0 \pm 4,6$ & \\
\hline \multicolumn{7}{|c|}{$\begin{array}{l}\text { Vacinação contra o tétano durante a } \\
\text { gravidez }\end{array}$} \\
\hline Sim & $43,8 \pm 16,8$ & 0,438 & $38,7 \pm 9,8$ & 0,456 & $27,2 \pm 9,6$ & 0,605 \\
\hline Não & $42,4 \pm 29,4$ & & $38,1 \pm 17,1$ & & $26,4 \pm 6,0$ & \\
\hline \multicolumn{7}{|l|}{ Tipo de parto } \\
\hline Normal & $44,3 \pm 16,6$ & 0,210 & $39,1 \pm 9,9$ & 0,148 & $26,5 \pm 6,3$ & 0,315 \\
\hline Cesárea & $41,4 \pm 18,8$ & & $36,9 \pm 10,6$ & & $25,9 \pm 4,9$ & \\
\hline
\end{tabular}

situação dos meninos do atual estudo, no $6^{\circ}$ mês, foi igualmente constatada em todos os períodos de outra coorte ${ }^{4}$. As diferenças segundo o sexo justificam-se, pois segundo características anatômicas e fisiológicas as meninas são de menor peso ao nascer e os meninos de maior estrutura corporal e tecido muscular ${ }^{16}$.
Em todos os intervalos de idade avaliados, a velocidade de comprimento foi maior nos casos de mães com estatura adequada, conforme outros achados ${ }^{21,22}$. A relação da estatura materna com a dos descendentes expressa a determinação biológica do crescimento infantil. O potencial genético de crescimento é influenciado pelas con- 
Tabela 3. Velocidade de peso de crianças até o sexto mês de vida segundo sexo, características maternas, cuidados durante a gravidez, variáveis de nascimento, segurança alimentar e nutricional, dificuldade para amamentar, práticas alimentares, uso de chupeta, presença de episódios de diarreia/vômito, saúde mental materna e vigilância do crescimento. Mamanguape, Paraíba, 2018.

\begin{tabular}{|c|c|c|c|c|c|c|}
\hline \multirow{3}{*}{ Variáveis } & \multicolumn{6}{|c|}{ Velocidade de peso (g/dia) } \\
\hline & \multicolumn{2}{|c|}{ Mês 1} & \multicolumn{2}{|c|}{ Mês 2} & \multicolumn{2}{|c|}{ Mês 6} \\
\hline & Média $\pm \mathrm{DP}$ & p-valor* & Média $\pm \mathrm{DP}$ & p-valor* & Média $\pm \mathrm{DP}$ & p-valor ${ }^{*}$ \\
\hline \multicolumn{7}{|l|}{ Peso ao nascer (g) } \\
\hline Satisfatório $(\geq 3.000)$ & $43,8 \pm 16,9$ & 0,490 & $38,1 \pm 9,7$ & 0,337 & $25,7 \pm 6,2$ & 0,268 \\
\hline Insuficiente (2.500-2.999) & $43,7 \pm 17,7$ & & $41,5 \pm 11,0$ & & $26,6 \pm 6,0$ & \\
\hline \multicolumn{7}{|l|}{ Segurança alimentar e nutricional } \\
\hline $\begin{array}{l}\text { Segurança alimentar/ } \\
\text { insegurança alimentar leve }\end{array}$ & $44,9 \pm 16,2$ & 0,044 & $39,8 \pm 9,5$ & 0,006 & $26,7 \pm 6,2$ & 0,100 \\
\hline $\begin{array}{l}\text { Insegurança alimentar } \\
\text { moderada/grave }\end{array}$ & $38,0 \pm 19,7$ & & $34,5 \pm 10,9$ & & $25,0 \pm 5,0$ & \\
\hline \multicolumn{7}{|l|}{ Dificuldade para amamentar } \\
\hline Não & $45,0 \pm 16,6$ & 0,047 & $38,8 \pm 10,0$ & 0,395 & $26,4 \pm 6,2$ & 0,516 \\
\hline Sim & $40,3 \pm 18,0$ & & $37,9 \pm 10,4$ & & $26,4 \pm 6,0$ & \\
\hline \multicolumn{7}{|l|}{ Tipo de aleitamento materno } \\
\hline Exclusivo/predominante & $44,4 \pm 15,3$ & 0,326 & $39,0 \pm 9,3$ & 0,374 & $27,9 \pm 7,6$ & 0,217 \\
\hline Misto/artificial & $43,1 \pm 18,8$ & & $38,4 \pm 10,7$ & & $26,3 \pm 5,9$ & \\
\hline \multicolumn{7}{|l|}{ Consumo de leite de vaca } \\
\hline Não & $44,0 \pm 17,3$ & 0,211 & $40,3 \pm 10,4$ & 0,711 & $26,6 \pm 5,9$ & 0,320 \\
\hline Sim & $38,3 \pm 8,0$ & & $38,6 \pm 10,0$ & & $26,0 \pm 6,5$ & \\
\hline \multicolumn{7}{|l|}{ Consumo de mingau } \\
\hline Não & $45,5 \pm 16,1$ & 0,013 & $39,6 \pm 9,5$ & 0,068 & $26,0 \pm 5,9$ & 0,738 \\
\hline $\operatorname{Sim}$ & $38,0 \pm 19,0$ & & $36,9 \pm 10,8$ & & $26,7 \pm 6,1$ & \\
\hline \multicolumn{7}{|l|}{ Consumo de água/chá } \\
\hline Não & $44,3 \pm 16,1$ & 0,377 & $39,2 \pm 10,4$ & 0,351 & $28,0 \pm 6,9$ & 0,322 \\
\hline $\operatorname{Sim}$ & $43,4 \pm 17,7$ & & $38,5 \pm 9,9$ & & $26,4 \pm 6,0$ & \\
\hline \multicolumn{7}{|l|}{ Consumo de fórmula infantil } \\
\hline Não & $45,1 \pm 15,7$ & 0,158 & $39,2 \pm 9,1$ & 0,291 & $27,1 \pm 5,2$ & 0,228 \\
\hline Sim & $42,2 \pm 18,4$ & & $38,2 \pm 11,0$ & & $26,2 \pm 6,3$ & \\
\hline \multicolumn{7}{|l|}{ Uso de chupeta } \\
\hline Não & $44,0 \pm 18,6$ & 0,432 & $39,4 \pm 9,0$ & 0,210 & $26,5 \pm 6,1$ & 0,442 \\
\hline Sim & $43,5 \pm 14,9$ & & $38,1 \pm 10,9$ & & $26,3 \pm 6,0$ & \\
\hline \multicolumn{7}{|l|}{ Diarreia/Vômito } \\
\hline Não & $44,1 \pm 17,7$ & 0,256 & $39,2 \pm 9,8$ & 0,555 & $26,9 \pm 5,5$ & 0,285 \\
\hline Sim & $41,2 \pm 11,1$ & & $38,7 \pm 13,3$ & & $26,2 \pm 6,2$ & \\
\hline \multicolumn{7}{|l|}{$\begin{array}{l}\text { Preenchimento do peso na } \\
\text { Caderneta de Saúde da Criança }\end{array}$} \\
\hline Sim & $47,9 \pm 19,7$ & 0,041 & $39,7 \pm 10,5$ & 0,135 & $27,4 \pm 4,7$ & 0,864 \\
\hline Não & $42,1 \pm 16,1$ & & $37,8 \pm 9,4$ & & $26,1 \pm 6,5$ & \\
\hline
\end{tabular}

\pm DP: desvio-padrão; ${ }^{\star}$ Teste T de Student.

Fonte: Elaborado pelos autores.

dições ambientais e socioeconômicas nas quais o indivíduo está inserido, representando a mãe uma importante interface entre a criança e o ambiente ${ }^{22,23}$.

A depressão materna pode gerar prejuízos na capacidade materna de amamentar, alimen- tar e cuidar do bebê, bem como no vínculo da mãe com o filho como um todo, com possíveis consequências negativas no crescimento e desenvolvimento da criança ${ }^{13,24,25}$, inclusive na atividade cerebral ${ }^{25}$. Em concordância, metanálises mostraram que as chances de déficits de peso e 
Tabela 4. Velocidade de comprimento de crianças até o sexto mês de vida segundo sexo, características maternas, cuidados durante a gravidez, variáveis de nascimento, segurança alimentar e nutricional, dificuldade para amamentar, práticas alimentares, uso de chupeta, presença de episódios de diarreia/vômito, saúde mental materna e vigilância do crescimento. Mamanguape, Paraíba, 2018.

\begin{tabular}{|c|c|c|c|c|c|c|}
\hline \multirow{3}{*}{ Variáveis } & \multicolumn{6}{|c|}{ Velocidade de comprimento (cm/mês) } \\
\hline & \multicolumn{2}{|c|}{ Mês 1} & \multicolumn{2}{|c|}{ Mês 2} & \multicolumn{2}{|c|}{ Mês 6} \\
\hline & Média \pm DP & p-valor* & Média \pm DP & p-valor* & Média \pm DP & p-valor ${ }^{*}$ \\
\hline \multicolumn{7}{|l|}{ Sexo da criança } \\
\hline Masculino & $5,8 \pm 2,1$ & 0,263 & $4,7 \pm 1,0$ & 0,059 & $3,0 \pm 0,4$ & 0,018 \\
\hline Feminino & $5,6 \pm 2,0$ & & $4,4 \pm 0,9$ & & $2,9 \pm 0,4$ & \\
\hline \multicolumn{7}{|l|}{ Idade materna (anos) } \\
\hline $18-34$ & $5,8 \pm 2,0$ & 0,053 & $4,6 \pm 1,0$ & 0,479 & $3,0 \pm 0,4$ & 0,196 \\
\hline$>34$ & $5,1 \pm 2,2$ & & $4,5 \pm 0,9$ & & $2,8 \pm 0,3$ & \\
\hline \multicolumn{7}{|l|}{ Estatura materna $(\mathrm{cm})$} \\
\hline Adequada & $5,9 \pm 2,1$ & 0,047 & $4,6 \pm 1,0$ & 0,039 & $3,0 \pm 0,4$ & 0,030 \\
\hline Baixa estatura $(<155)$ & $5,0 \pm 2,0$ & & $4,3 \pm 1,0$ & & $2,9 \pm 0,4$ & \\
\hline \multicolumn{7}{|c|}{ Transtorno Mental Comum materno } \\
\hline Não & $5,7 \pm 2,6$ & 0,656 & $4,7 \pm 0,8$ & 0,153 & $3,0 \pm 0,4$ & 0,332 \\
\hline Sim & $5,5 \pm 2,0$ & & $4,5 \pm 1,1$ & & $3,0 \pm 0,4$ & \\
\hline \multicolumn{7}{|c|}{ Transtorno Mental Grave materno } \\
\hline Não & $5,7 \pm 2,2$ & 0,507 & $4,6 \pm 0,8$ & 0,392 & $3,0 \pm 0,4$ & 0,429 \\
\hline Sim & $5,7 \pm 2,1$ & & $4,5 \pm 1,0$ & & $3,0 \pm 0,4$ & \\
\hline \multicolumn{7}{|l|}{ Início do pré-natal } \\
\hline $1^{\circ}$ trimestre & $5,7 \pm 2,0$ & 0,306 & $4,6 \pm 1,0$ & 0,396 & $3,0 \pm 0,4$ & 0,390 \\
\hline Após $1^{\circ}$ trimestre & $5,5 \pm 2,6$ & & $4,5 \pm 1,0$ & & $2,9 \pm 0,3$ & \\
\hline \multicolumn{7}{|l|}{$\mathrm{N}^{\circ}$ de consultas de pré-natal } \\
\hline$\geq 6$ & $5,7 \pm 1,9$ & 0,413 & $4,6 \pm 1,0$ & 0,295 & $3,0 \pm 0,4$ & 0,427 \\
\hline$<6$ & $5,6 \pm 2,8$ & & $4,4 \pm 1,1$ & & $2,9 \pm 0,4$ & \\
\hline \multicolumn{7}{|c|}{$\begin{array}{l}\text { Ingestão de bebida alcoólica durante } \\
\text { a gravidez }\end{array}$} \\
\hline Não & $5,8 \pm 2,1$ & 0,118 & $4,6 \pm 1,0$ & 0,127 & $3,0 \pm 0,4$ & 0,111 \\
\hline Sim & $4,9 \pm 1,4$ & & $4,1 \pm 1,1$ & & $2,8 \pm 0,4$ & \\
\hline \multicolumn{7}{|l|}{ Fumo durante a gravidez } \\
\hline Não & $5,7 \pm 2,1$ & 0,595 & $4,5 \pm 1,0$ & 0,595 & $3,0 \pm 0,4$ & 0,771 \\
\hline Sim & $5,9 \pm 1,5$ & & $4,6 \pm 0,9$ & & $3,1 \pm 0,4$ & \\
\hline \multicolumn{7}{|c|}{$\begin{array}{l}\text { Vacinação contra o tétano durante a } \\
\text { gravidez }\end{array}$} \\
\hline Sim & $5,8 \pm 2,0$ & 0,001 & $4,6 \pm 1,0$ & 0,094 & $3,0 \pm 0,4$ & 0,179 \\
\hline Não & $2,6 \pm 0,3$ & & $3,9 \pm 1,2$ & & $2,8 \pm 0,2$ & \\
\hline \multicolumn{7}{|l|}{ Tipo de parto } \\
\hline Normal & $5,9 \pm 2,1$ & 0,708 & $4,7 \pm 1,0$ & 0,859 & $3,0 \pm 0,4$ & 0,543 \\
\hline Cesárea & $5,7 \pm 2,1$ & & $4,5 \pm 1,1$ & & $3,0 \pm 0,5$ & \\
\hline
\end{tabular}

estatura na criança aumentam no caso de mães deprimidas ${ }^{24}$, obtendo-se achados similares para a velocidade de peso no $1^{\circ}$ mês entre as crianças acompanhadas nesta coorte. Adicionalmente, o estudo em tela apontou resultado análogo para o perímetro cefálico nos três momentos do seguimento, cuja relevância deriva da carência de 
Tabela 4. Velocidade de comprimento de crianças até o sexto mês de vida segundo sexo, características maternas, cuidados durante a gravidez, variáveis de nascimento, segurança alimentar e nutricional, dificuldade para amamentar, práticas alimentares, uso de chupeta, presença de episódios de diarreia/vômito, saúde mental materna e vigilância do crescimento. Mamanguape, Paraíba, 2018.

\begin{tabular}{|c|c|c|c|c|c|c|}
\hline \multirow{3}{*}{ Variáveis } & \multicolumn{6}{|c|}{ Velocidade de comprimento $(\mathrm{cm} / \mathrm{mês})$} \\
\hline & \multicolumn{2}{|c|}{ Mês 1} & \multicolumn{2}{|c|}{ Mês 2} & \multicolumn{2}{|c|}{ Mês 6} \\
\hline & Média \pm DP & p-valor* & Média $\pm \mathrm{DP}$ & p-valor* & Média \pm DP & p-valor ${ }^{*}$ \\
\hline \multicolumn{7}{|l|}{ Peso ao nascer $(\mathrm{g})$} \\
\hline Satisfatório $(\geq 3.000)$ & $5,6 \pm 2,0$ & 0,721 & $4,5 \pm 1,0$ & 0,984 & $2,9 \pm 0,4$ & 0,952 \\
\hline Insuficiente $(2.500-2.999)$ & $5,9 \pm 2,3$ & & $4,9 \pm 1,0$ & & $3,1 \pm 0,4$ & \\
\hline \multicolumn{7}{|l|}{ Segurança alimentar e nutricional } \\
\hline $\begin{array}{l}\text { Segurança alimentar/insegurança } \\
\text { alimentar leve }\end{array}$ & $5,7 \pm 2,1$ & 0,654 & $4,5 \pm 1,0$ & 0,696 & $3,0 \pm 0,4$ & 0,727 \\
\hline $\begin{array}{l}\text { Insegurança alimentar moderada/ } \\
\text { grave }\end{array}$ & $5,8 \pm 2,0$ & & $4,6 \pm 0,9$ & & $3,0 \pm 0,3$ & \\
\hline \multicolumn{7}{|l|}{ Dificuldade para amamentar } \\
\hline Não & $6,0 \pm 2,0$ & 0,002 & $4,6 \pm 1,0$ & 0,042 & $3,0 \pm 0,4$ & 0,251 \\
\hline Sim & $4,8 \pm 2,0$ & & $4,2 \pm 1,0$ & & $2,9 \pm 0,4$ & \\
\hline \multicolumn{7}{|l|}{ Tipo de aleitamento materno } \\
\hline Exclusivo/predominante & $5,7 \pm 1,8$ & 0,673 & $4,6 \pm 0,9$ & 0,272 & $2,9 \pm 0,3$ & 0,676 \\
\hline Misto/artificial & $5,6 \pm 2,3$ & & $4,5 \pm 1,0$ & & $3,0 \pm 0,4$ & \\
\hline \multicolumn{7}{|l|}{ Consumo de leite de vaca } \\
\hline Não & $5,7 \pm 2,1$ & 0,085 & $4,1 \pm 1,0$ & 0,065 & $3,0 \pm 0,4$ & 0,628 \\
\hline Sim & $4,6 \pm 1,8$ & & $4,6 \pm 1,0$ & & $3,0 \pm 0,4$ & \\
\hline \multicolumn{7}{|l|}{ Consumo de mingau } \\
\hline Não & $5,6 \pm 2,0$ & 0,288 & $4,6 \pm 1,0$ & 0,092 & $3,0 \pm 0,4$ & 0,433 \\
\hline Sim & $5,5 \pm 2,4$ & & $4,4 \pm 1,1$ & & $3,0 \pm 0,4$ & \\
\hline \multicolumn{7}{|l|}{ Consumo de água/chá } \\
\hline Não & $5,8 \pm 1,8$ & 0,282 & $4,6 \pm 1,0$ & 0,605 & $3,1 \pm 0,6$ & 0,303 \\
\hline Sim & $5,6 \pm 2,3$ & & $4,6 \pm 1,0$ & & $3,0 \pm 0,4$ & \\
\hline \multicolumn{7}{|l|}{ Consumo de fórmula infantil } \\
\hline Não & $5,7 \pm 2,0$ & 0,516 & $4,6 \pm 1,0$ & 0,255 & $3,0 \pm 0,3$ & 0,458 \\
\hline Sim & $5,7 \pm 2,2$ & & $4,5 \pm 1,0$ & & $3,0 \pm 0,4$ & \\
\hline \multicolumn{7}{|l|}{ Uso de chupeta } \\
\hline Não & $5,8 \pm 2,0$ & 0,288 & $4,7 \pm 0,9$ & 0,020 & $3,0 \pm 0,4$ & 0,230 \\
\hline Sim & $5,6 \pm 2,3$ & & $4,4 \pm 1,0$ & & $2,9 \pm 0,3$ & \\
\hline \multicolumn{7}{|l|}{ Diarreia/Vômito } \\
\hline Não & $5,9 \pm 1,1$ & 0,678 & $4,6 \pm 1,0$ & 0,078 & $3,0 \pm 0,4$ & 0,142 \\
\hline Sim & $5,7 \pm 2,2$ & & $4,1 \pm 1,2$ & & $2,9 \pm 0,4$ & \\
\hline \multicolumn{7}{|l|}{$\begin{array}{l}\text { Preenchimento do comprimento na } \\
\text { Caderneta de Saúde da Criança }\end{array}$} \\
\hline Sim & $5,7 \pm 2,1$ & 0,682 & $5,1 \pm 0,8$ & 0,104 & $3,1 \pm 0,4$ & 0,203 \\
\hline Não & $5,3 \pm 2,7$ & & $4,5 \pm 1,0$ & & $3,0 \pm 0,4$ & \\
\hline
\end{tabular}

\pm DP: desvio-padrão; ${ }^{\star}$ Teste T de Student.

Fonte: Elaborado pelos autores.

lacional ${ }^{27}$. A assistência pré-natal é fundamental para proteger a saúde obstétrica e prevenir a ocorrência de eventos adversos no crescimento, desenvolvimento e saúde do recém-nascido ${ }^{23,28}$.

A ingestão de bebida alcoólica durante a gestação influenciou o ganho de perímetro cefálico nos dois primeiros momentos da coorte. Déficits no crescimento e desenvolvimento fetal decorrentes da ingestão de álcool na gravidez relacionam-se à facilidade do etanol atravessar a barreira placentária entre o sangue e o cérebro e de produzir alterações estruturais e/ou funcionais no 
sistema nervoso central ${ }^{29}$. A despeito disso, sendo sobremodo escassa a literatura que desenvolve o assunto, vale destacar um estudo descritivo conduzido na Austrália que conferiu à desordem do espectro alcoólico fetal como causa principal de microcefalia em nascimentos aborígenes ${ }^{30}$.

Apesar de não existir relatos similares na literatura da associação verificada para a vacinação contra o tétano durante a gravidez com as velocidades de perímetro cefálico, nos três momentos, e de comprimento, no mês 1, é possível cogitar que esse resultado esteja circunstanciado ao fato do tétano neonatorum acarretar dificuldade progressiva na alimentação, como na sucção e deglutição, que redunda em fome. No recém-nascido, a entidade é ocasionada pela insuficiência de anticorpos maternos transferidos por via transplacentária, cuja principal forma de prevenção é a vacinação durante a gestação ${ }^{31}$.

Nesse estudo foi possível observar que o parto normal contribuiu com maiores ganhos de perímetro cefálico nas duas primeiras observações após o nascimento da criança. Esse tipo de parto constitui uma via de nascimento que facilita o primeiro contato da díade e possibilita melhores condições para o aleitamento materno no período pós-parto imediato ${ }^{4,32}$, como mostrado em estudo desenvolvido no Rio de Janeiro em relação à amamentação na primeira hora de vida ${ }^{32} \mathrm{e}$ por meio de uma coorte de prematuros na qual o nascimento por cessaria influenciou negativamente a produção de leite materno como consequência da lactogenese tardia ${ }^{33}$.

No presente estudo, o maior ganho de peso, no $1^{\circ}$ e $2^{\circ}$ mês, constatado nas crianças em situação de segurança alimentar/insegurança alimentar leve tem respaldos na literatura ${ }^{34-36}$. As vias elucidativas dessa relação incluem os efeitos da insegurança alimentar e nutricional, principalmente, na quantidade e/ou qualidade da alimentação ${ }^{34-36}$, bem quanto em outros aspectos como na nutrição e saúde mental da mãe, no início e na duração da amamentação, e no sistema imunológico e suscetibilidade a processos infecciosos da criança ${ }^{36}$. Essas premissas sugerem-se também aplicáveis para as menores médias na velocidade de perímetro cefálico, durante toda a coorte, quando a presença de insegurança alimentar moderada/grave, cujos achados são inéditos ${ }^{35,36}$.

Como em outras coortes nacionais ${ }^{4,33}$ e internacionais ${ }^{8,37,38}$, os parâmetros antropométricos do presente estudo mostraram associação com o relato de dificuldade na amamentação e com práticas alimentares inadequadas (aleitamento materno misto/artificial e consumo de mingau e de fórmula infantil). O aleitamento materno quando obstaculizado pode afetar a produção de leite materno e levar à sua interrupção de forma precoce, da mesma forma que a complementação da amamentação com outros líquidos contribui sobremaneira com o desmame precoce ${ }^{4,39}$. Fórmulas industrializadas introduzidas precocemente podem desencadear quadros infecciosos, enquanto o leite materno contém os nutrientes essenciais para o crescimento e desenvolvimento do lactente ${ }^{39}$.

No $2^{\circ}$ mês de vida da criança, a associação observada entre o uso de chupeta e menores ganhos de comprimento pode ter relação com o fato desse objeto induzir a posicionamento incorreto da língua da criança no seio da mãe e a inadequada sucção do leite materno. Desse modo, a chupeta aumenta a possibilidade de desmame antecipa$\mathrm{do}^{39,40}$.

Na Austrália, pesquisadores mostraram que a prevalência de microcefalia associou-se de maneira significativa com condições infecciosas ${ }^{29}$, com analogia neste trabalho ao se discriminar a presença de episódios de diarreia/vômito como determinante da velocidade de perímetro cefálico no $1^{\circ}$ primeiro mês de vida. Como ideia subliminar é possível argumentar que em decorrência da substituição do leite materno por outros alimentos de menor valor nutricional e/ou contaminados, há uma maior vulnerabilidade relacionada ao desenvolvimento de processos infecciosos, com consequências negativas no crescimento ${ }^{5}$.

O preenchimento das curvas de crescimento da CSC foi avaliado neste estudo com o propósito de verificar se ganhos antropométricos positivos estariam relacionados com maior adequação, evidenciando que o instrumento cumpre seu papel na vigilância e promoção do estado nutricional da criança. Essa hipótese pôde ser confirmada em algum momento do seguimento para o perímetro cefálico e o peso do lactente, contrariando os resultados obtidos por meio de um estudo transversal prévio ${ }^{41}$. Esses achados, ao serem pioneiros na literatura, reforçam que a inadequada utilização da CSC inviabiliza o diagnóstico do estado nutricional, a identificação de fatores de risco e a vigilância do crescimento ${ }^{23}$.

\section{Conclusões}

O método de avaliação longitudinal utilizado nesse estudo propiciou a identificação de fatores, a maioria deles modificáveis, que influenciaram a velocidade de crescimento de lactentes durante 
os primeiros seis meses de vida. A relevância dos achados está atrelada à avaliação da velocidade de crescimento em uma fase crítica do desenvolvimento, possibilitando a identificação precoce dos determinantes desse processo. Como limitação podem ser citadas as perdas de seguimento. Porém, a análise de perda diferencial conforme o acompanhamento completo ou não (perdas de seguimento) da criança mostrou que não houve diferença entre os dois grupos, inferindo-se que os resultados apresentados não foram comprometidos por viés de seleção.

A análise de fatores de risco da velocidade de crescimento em três momentos diferentes (do período do nascimento até os seis meses de vida) realizada nesse estudo permitiu identificar a influência, em algum dos indicadores e no mínimo em um momento, da baixa estatura materna, da presença de transtorno mental grave na mãe, de cuidados pré-natais inadequados, do parto cesáreo, da insegurança alimentar moderada/grave familiar, da dificuldade materna em amamentar, do aleitamento materno misto/artificial, da introdução precoce de mingau e de fórmula infantil, do uso de chupeta, da presença de diarreia/ vômito e do não preenchimento das curvas de crescimento da CSC. Esses fatores são, na maioria, modificáveis e têm em comum a relação com o aleitamento materno do lactente. Os achados supracitados indicam a necessidade de intervenções que visem a qualidade do pré-natal e de cuidados adequados durante a gestação, o incentivo ao parto natural, a orientação materna sobre práticas alimentares adequadas até o $6^{\circ}$ mês de vida e em relação aos prejuízos do relacionados ao uso de chupeta, a preocupação com o preenchimento das curvas de crescimento da CSC, e a promoção da saúde materno-infantil.

\section{Colaboradores}

D Figueroa Pedraza participou da elaboração do protocolo de estudo, concepção do artigo, análise e interpretação dos dados, redação e revisão final do artigo. ACL Lins participaram da análise e interpretação dos dados, redação e revisão final do artigo. 


\section{Referências}

1. Arimatea JE, Silva CMFP, Costa AJL, Fonseca SC, Gama SGN, Lacerda EMA, Kale PL. Low birthweight and postnatal weight in full-term infants under six months old, Rio de Janeiro, RJ, Brazil. Cien Saude Colet 2015; 20(5):1459-1466.

2. Clemente MG, Capobianco G, Galasso PM, Dessole F, Virdis G, Sanna MG, Olzai MG, Argiolas L, Dessole S, Antonucci R. Postnatal Growth in a Cohort of Sardinian Intrauterine Growth-Restricted Infants. BioMed Res Int 2017; 2017:9382083.

3. Avan BI, Raza SA, Kirkwood BR. An epidemiological study of urban and rural children in Pakistan: examining the relationship between delayed psychomotordevelopment, low birth weight and postnatal growth failure. Trans R Soc Trop Med Hyg 2015; 109(3):189196.

4. Fonseca PCA, Carvalho CA, Ribeiro SAV, Nobre LN, Pessoa MC, Ribeiro AQ, Priore SE, Franceschini SCC. Determinantes da velocidade média de crescimento de crianças até seis meses de vida: um estudo de coorte. Cien Saude Colet 2017; 22(8):2713-2726.

5. Vieira SA, Magalhães TCA, Ribeiro AQ, Priore SE, Franceschini SCC, Sant'Ana LFR. Fatores associados às velocidades de ganho de peso e de comprimento nos primeiros seis meses de vida. Cad Saude Colet 2015; 23(3):309-315

6. Fonseca PCA, Carvalho VA, Carvalho CA, Ribeiro AQ, Priore SE, Franceschini SCC, Vieira SA. Efeito do tabagismo na gestação sobre o excesso de peso e déficit de crescimento em crianças nos primeiros seis meses de vida: uma análise de sobrevida. Rev Bras Saude Matern Infant 2018; 18(2):371-379.

7. World Health Organization (WHO). Indicators for assessing infant and young child feeding practices: conclusions of a consensus meeting held 6-8 November 2007 in Washington D.C., USA. Genebra: WHO; 2008.

8. Hosseini SM, Maracy MR, Sarrafzade S, Kelishadi R. Child weight growth trajectory and its determinants in a sample of Iranian children from birth until 2 years of age. Int J Prev Med 2014; 5(3):348-355.

9. Ballard TJ, Kepple AW, Cafiero C. The food insecurity experience scale: developing a global standard for monitoring hunger worldwide. Technical Paper. Roma: FAO; 2013.

10. Centers for Disease Control and Prevention (CDC). National Center for Health Statistic. Growth Curves [Internet]. 2000 [acessado 2018 set 18]. Disponível em: http://www.cdc.gov/growthcharts/cdc_charts.htm.

11. Brasil. Ministério da Saúde (MS). Saúde da criança: aleitamento materno e alimentação complementar. Brasília: MS; 2015.

12. Mari JJ, Williams PA comparison of the validity of two psychiatric screening questionnaires (GHQ-12 and SRQ-20) in Brazil, using Relative Operating Characteristic (ROC) analysis. Psychol Med 1985; 15(3):651659.

13. Hassan BK, Werneck GL, Hasselmann MH. Saúde mental materna e estado nutricional de crianças aos seis meses de vida. Rev Saude Publica 2016; 50:7.

14. Onis M, Onyango AW, Van den Broeck J, Chumlea WC, Martorell R. Measurement and standardization protocols for anthropometry used in the construction of a new international growth reference. Food Nutr Bull 2004; 25(Supl. 1):15-27.
15. Puffer RR, Serrano CV. Patterns of birth weight. Washington, D.C.: PAHO; 1987.

16. Jaldin MGM, Pinheiro FS, Santos AM, Muniz NC Crescimento infantil comparado com as referências NCHS e o padrão WHO/2006. Rev Nutr 2013; 26(1):17-26.

17. Macchiaverni LML, Barros Filho AA. Perímetro cefálico: por que medir sempre. Med 1998; 31:595-609.

18. Onis M, Onyango AW. The Centers for Disease Control and Prevention 2000 growth charts and the growth of breastfed infants. Acta Paediatr 2003; 92:413-419.

19. Jaldin MGM, Pinheiro FS, Santos AM, Muniz NC, Brito LMO. Crescimento do perímetro cefálico nos primeiros seis meses em crianças em aleitamento materno exclusivo. Rev Paul Pediatr 2011; 29(4):509-514.

20. Amorim MST, Melo NA. Revisiting head circumference of Brazilian newborns in public and private maternity hospitals. Arq Neuropsiquiatr 2017; 75(6):372380.

21. Abdulahi A, Shab-Bidar S, Rezaei S, Djafarian K. Nutritional Status of Under Five Children in Ethiopia: A Systematic Review and Meta-Analysis. Ethiop J Health Sci 2017; 27(2):175-188.

22. Queiroz VAO, Assis AMO, Pinheiro SMC, Ribeiro Junior HC. Predictors of linear growth in the first year of life of a prospective cohort of full term children with normal birth weight. J Pediatr 2012; 88(1):79-86

23. Figueroa Pedraza D, Sales MC, Menezes TN. Fatores associados ao crescimento linear de crianças socialmente vulneráveis do Estado da Paraíba, Brasil. Cien Saude Colet 2016; 21(3):935-945.

24. Surkan PJ, Kennedy CA, Hurley KM, Black MM Maternal depression and early childhood growth in developing countries: systematic review and meta-analysis. Bull WHO 2011; 89(8):607-615.

25. Leivas PHS, Tejada CAO, Bertoldi AD, Santos AMA, Jacinto PA. Associação da posição socioeconômica e da depressão materna com a saúde das crianças: avaliação da PNAD 2008, Brasil. Cien Saude Colet 2018; 23(5):1635-1645

26. Brentani A, Fink G. Maternal depression and child development: Evidence from São Paulo's Western Region Cohort Study. Rev Assoc Med Bras 2016; 62(6):524-529.

27. Miglioli TC, Fonseca VM, Gomes Junior SC, Silva KS, Lira PIC, Batista Filho M. Fatores associados ao estado nutricional de crianças menores de cinco anos. Rev Saude Publica 2015; 49:59.

28. Nunes JT, Gomes KRO, Rodrigues MTP, Mascarenhas MDM. Qualidade da assistência pré-natal no Brasil: revisão de artigos publicados de 2005 a 2015. Cad Saude Colet 2016; 24(2):252-261.

29. Mesquita MA. Efeitos do álcool no recém-nascido. Einstein 2010; 8(3 Pt. 1):368-375.

30. Hansen M, Armstrong PK, Bower C, Batnam GS. Prevalence of microcephaly in an Australian population -based birth defects register, 1980 e 2015. Med J Aust 2017; 206(8):351-356.

31. Gomes AP, Freitas BAC, Rodrigues DC, Silveira GL, Tavares W, Siqueira-Batista R. Infecção por Clostridium tetani no recém-nascido: revisão sobre o tétano neonatorum. Rev Bras Ter Inten 2011; 23(4):484-491. 
32. Pereira CRVR, Fonseca VM, Oliveira MIC, Souza IEO, Mello RR. Avaliação de fatores que interferem na amamentação na primeira hora de vida. Rev Bras Epidemiol 2013; 16(2):525-534.

33. Méio MDBB, Villela LDV, Gomes Júnior SCS, Tovar CM, Moreira MEL. Amamentação em lactentes nascidos pré-termo após alta hospitalar: acompanhamento durante o primeiro ano de vida. Cien Saude Colet 2018; 23(7):2403-2412.

34. Santos LP, Gigante DP. Relação entre insegurança alimentar e estado nutricional de crianças brasileiras menores de cinco anos. Rev Bras Epidemiol 2013; 16(4):984-994.

35. Morais DC, Dutra LV, Franceschini SCC, Priore SE. Insegurança alimentar e indicadores antropométricos, dietéticos e sociais em estudos brasileiros: uma revisão sistemática. Cien Saude Colet 2014; 19(5):1475-1488.

36. Maitra C. A review of studies examining the link between food insecurity and malnutrition. Technical Paper. Roma: FAO; 2018.

37. Donma MM, Donma O. The influence of feeding patterns on head circumference among Turkish infants during the first 6 months of life. Brain Develop 1997; 19:393-397.

38. Johnson L, Van Jaarsveld CHM, Llewellyn CH, Cole TJ, Wardle J. Associations between infant feeding and the size, tempo and velocity of infant weight gain: SITAR analysis of the Gemini twin birth cohort. Int $J$ Obes 2014; 38:980-987.
39. Moraes BA. Fatores associados à interrupção do aleitamento materno exclusivo em lactentes com até 30 dias. Rev Gaucha Enferm 2016; 37(n. esp.):e2016-0044.

40. Carvalho CA, Fonsêca PCA, Nobre LN, Silva MA, Pessoa MC, Ribeiro AQ, Priore SE, Franceschini SCC. Fatores sociodemográficos, perinatais e comportamentais associados aos tipos de leite consumidos por crianças menores de seis meses: coorte de nascimento. Cien Saude Colet 2017; 22(11):3699-3709.

41. Palombo CNT, Duarte LS, Fujimori E, Toriyama TM. Uso e preenchimento da caderneta de saúde da criança com foco no crescimento e desenvolvimento. Rev Esc Enferm USP 2014; 48(n. esp.):60-67.

Artigo apresentado em 07/12/2019

Aprovado em 18/09/2020

Versão final apresentada em 20/09/2020

Editores-chefes: Romeu Gomes, Antônio Augusto Moura da Silva 